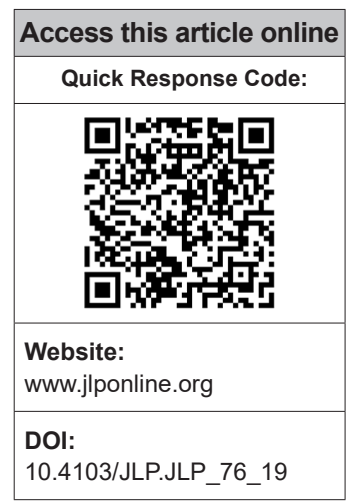

DOI:

10.4103/JLP.JLP_76_19
Departments of Pathology and ${ }^{1}$ Endocrinology, IPGME and R, Kolkata, West Bengal, India

Address for correspondence: Dr. Chhanda Das,

31 Eastern Park, First Road, Santoshpur, Kolkata - 700075 , West Bengal, India. E-mail: chhhdas@gmail.

Submission: 30-04-2019 Accepted: 16-11-2019

\title{
Evaluation of prognostic utility of Ki-67, P53, and 0-6-methylguanine-DNA methyltransferase expression in pituitary tumors
}

\author{
Chhanda Das, Pratap Mondal, Madhumita Mukhopadhyay, \\ Satinath Mukhopadhyay ${ }^{1}$, Ipsita Ghosh ${ }^{1}$, Anusha Handral ${ }^{1}$
}

\section{Abstract:}

BACKGROUND OR CONTEXT: Pituitary adenoma (PA) is the most common pathology of the pituitary gland. Pituitary tumors were historically considered benign, however, from recent advances in pathological and molecular analyses, numerous prognostic markers have been identified, allowing a better characterization of tumor behavior and prediction of response to treatment and recurrences.

AIMS AND OBJECTIVES: Evaluation of the epidemiological occurrence of pituitary tumors in our center and prediction of the benign, aggressive, or malignant nature of the tumor with the help of immunohistochemical markers (IHC) Ki-67, P53, and O-6-methylguanine-DNA methyltransferase (MGMT) along with radiology.

MATERIALS AND METHODS: A prospective study was done in 33 cases. Patients with clinically suspected pituitary tumors and related symptoms and signs are referred from the endocrine outpatient department and subsequently operated at the neurosurgery department were selected. We have studied the clinical features, radiology, histopathology, and IHC with the help of Ki-67, P53, and MGMT of PA over 2 years.

RESULTS: We have $94 \%$ (31/33) cases of PA among them $94 \%$ (29/31) cases are macroadenoma. The IHC was conducted on 30 cases (excluding 1 case of pituitary apoplexy) where Ki-67, p53, and MGMT have been used for IHC in order to analyze the prognosis of the PA, irrespective of the immunological subtype of the PA. In our study, only 13\% (4 patients) had MGMT score 0 and 2 patients, among these 4 patients having above cutoff level of Ki-67 and p53 value, considered as aggressive (in case of $\mathrm{Ki}-67>3 \%$ and $>50 \%$ in case of p53). When comparing MGMT expression with recurrence, a high degree of significance was found (Mann-Whitney U-test, $P=0.0038$ ). Most of the recurrent tumors (6/9) had MGMT score 1 or below and most of the nonrecurring tumor had MGMT score 2 or above. When comparing MGMT expression with aggressiveness, a high degree of significance was found (Mann-Whitney U-test, $P<0.0001$ ). Finally, combining the radiological $\mathrm{Ki}-67, \mathrm{p53}$, and MGMT values, two cases of aggressive adenoma have been seen in our study, the remaining being benign adenomas (WHO classification 2004). We did not encounter any case of pituitary carcinoma. MGMT did not show any significant correlation with radiological grading and histology.

CONCLUSION: The benign, aggressive, or malignant nature of PA can be effectively predicted with the help of IHC, such as Ki-67, p53, and MGMT. This helps in better patient management and predicts recurrences and prognosis.

Key words:

Immunohistochemistry, Ki-67, O-6-methylguanine-DNA methyltransferase, P53

This is an open access journal, and articles are distributed under the terms of the Creative Commons Attribution-NonCommercial-ShareAlike 4.0 License, which allows others to remix, tweak, and build upon the work non-commercially, as long as appropriate credit is given and the new creations are licensed under the identical terms.

For reprints contact: reprints@medknow.com
How to cite this article: Das $C$, Mondal $P$, Mukhopadhyay M, Mukhopadhyay S, Ghosh I, Handral A. Evaluation of prognostic utility of Ki-67, P53, and O-6-methylguanine-DNA methyltransferase expression in pituitary tumors. J Lab Physicians 2019;11:323-9. 


\section{Introduction}

Pituitary adenomas (PAs) constituting approximately $15 \%-20 \%$ of primary intracranial neoplasms and are the third most frequent histological type next by gliomas and meningiomas. ${ }^{[1]}$ PAs are classified clinically into the functioning and nonfunctioning adenomas, according to whether or not an endocrine syndrome is present. ${ }^{[2]}$ PRL-omas comprise nearly $80 \%$ of functioning pituitary tumors and about $40 \%-50 \%$ of all PA. ${ }^{[3]}$ Usually, endocrinologically functional tumors are often small and nonfunctioning tumors are large mostly present with mass effects. Most of these tumors are noninvasive and benign, and remain either within the sella or exhibit slow expansive growth displacing surrounding tissues. Invasiveness is defined as extension into sellar floor bone, the cavernous sinus, and/or the diaphragm sella, assessed on preoperative neuroimaging studies. So-called "invasive," PAs are not considered malignant by current definition, display benign behavior even in the presence of marked dural invasion. Truly, malignant pituitary tumors (pituitary carcinomas) are only defined by the presence of cerebrospinal or systemic metastases and are exceedingly rare with an incidence of $0.2 \%$ of symptomatic pituitary tumors. ${ }^{[4]}$ The so-called "aggressive" adenomas lie between benign adenomas and malignant pituitary carcinomas and display a rather distinct clinical behavior with marked/gross invasion of nearby anatomical structures and a tendency toward resistance to conventional treatments and early postoperative recurrence..$^{[4]}$ In 2004, the WHO published a classification system for pituitary tumors based upon immunohistochemistry identified existence of atypical adenomas as tumors morphological features of an "aggressive behaviour," substantiated by the presence of invasive growth, high mitotic index, a Ki-67 labeling index (LI) $>3 \%$ as well as extensive nuclear staining for p53. ${ }^{[5]}$ The DNA repair protein O-6-methylguanine-DNA methyltransferase (MGMT) is a key player in tumor cell resistance. Promoter methylation of MGMT activity and immunohistochemistry are used for determining the MGMT status. Whether MGMT promoter methylation of the pretreatment tumor predicts the MGMT status of recurrences, it is still now unclear. To address these questions, we want to determine MGMT activity immunoreactivity in pretreatment and recurrent pituitary tumor. In our study, we aimed to predict the benign, aggressive, or malignant nature of the tumor with the help of immunohistochemical markers MGMT, Ki-67, and P53.

\section{Materials and Methods}

This is a prospective and observational study conducted over a span of 18 months from January 2016 to June 2017 (with a total of 33 cases). The patients attending the endocrine outpatient department (OPD) and Neurosurgery OPD in our hospital and undergoing clinical and radiological evaluation with evidence of pituitary tumor and selected for operation were included in the study. Cytological and histopathological studies were done for confirmation of diagnosis and IHC studies were done to know Ki-67 LI and P53 value and MGMT immunohistochemical status. It was performed on 4-5 $\mu \mathrm{m}$ thick, formalin-fixed, paraffin-embedded tissue sections that were mounted on poly-1-lysine precoated slides. In histological sections, the different patterns of acidophilic to chromophobic color of the cytoplasm [Figures 1-3]. Interpretation of immunostains: Evaluated by scoring system depending on distribution (homogenous/ heterogenous), intensity and percentage of positive cells. Counting was done at high-power magnification $(\times 400)$. For all cases, counting was done without knowing the type of adenoma. The lowest MGMT score "0" indicates no staining, and the highest score " 5 " indicates homogenous distribution with $>50 \%$ of cell positivity ${ }^{[6]}[$ Table 1]. This antibody stains both nucleus and cytoplasm in positive cells in formalin-fixed paraffin-embedded tissue sections [Figure 4]. The MIB-1 LI (Ki-67) was calculated by calculating the percentage of labeled nuclei per 1000 nucleated cells in an area with maximum population of cells. Counting was done at high-power magnification $(\times 400)$. Ki-67 LI $<3 \%$ is taken to be noninvasive adenoma. Areas of necrosis, normal adenohypophysial cells and endothelial cells were excluded from the evaluation. This antibody stains nucleus in positive cells in formalin-fixed paraffin-embedded tissue sections [Figure 5]. In P53 value, cell positivity was counted by calculating the percentage of labeled nuclei per 1000 nucleated cells in an area with maximum population of cells. The expression of p53 was graded semi-quantitatively in three categories: severe (when most of the cells are positive), moderate (when approximately $50 \%$ of the cells are

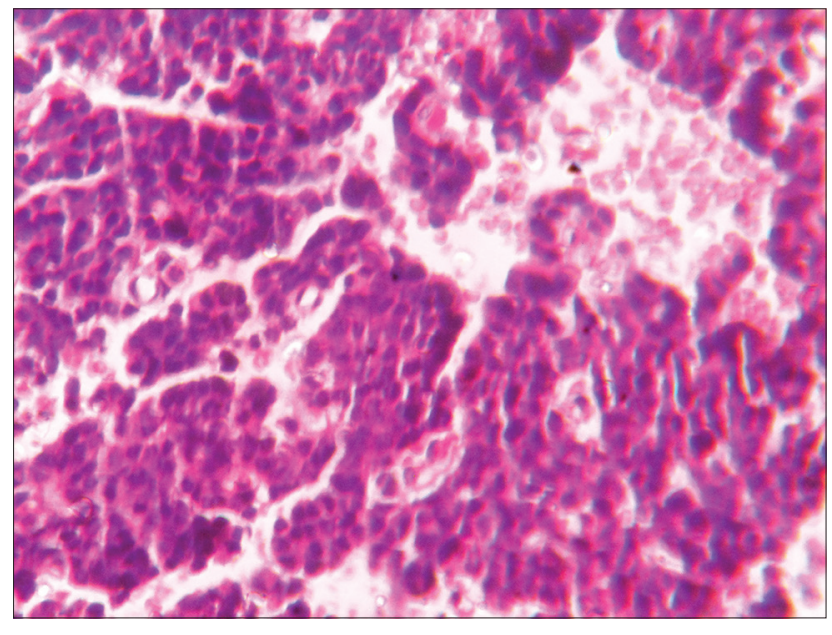

Figure 1: Basophilic pituitary adenoma $(\times 400)$ 


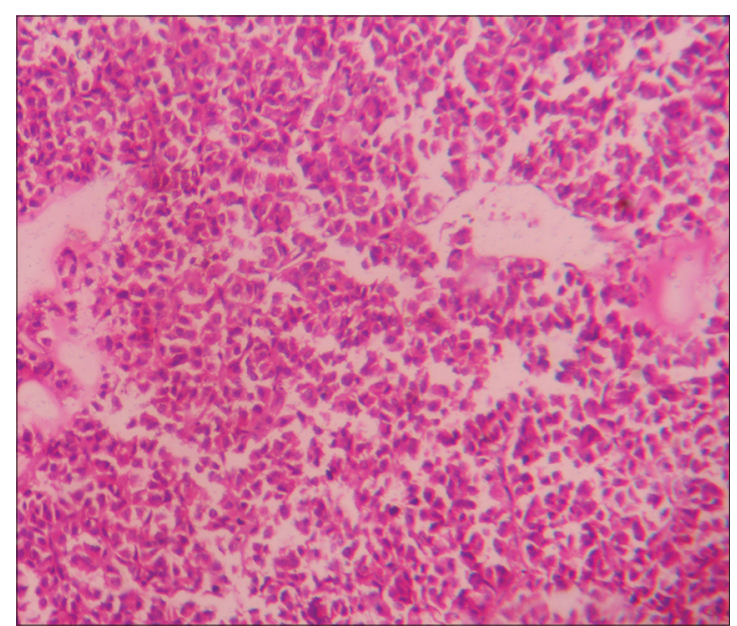

Figure 2: Eosinophilic adenoma $(\times 400)$

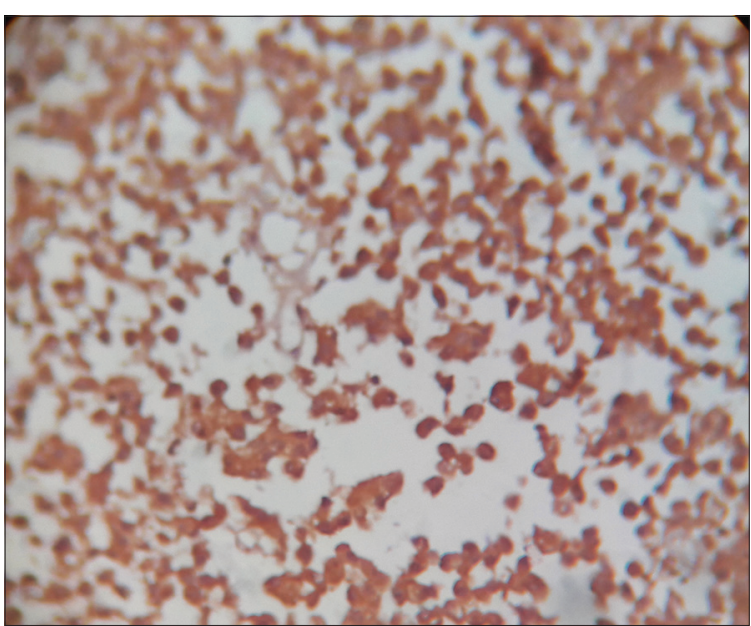

Figure 4: 0-6-methylguanine-DNA methyltransferase positive stain $\times 400$

positive), and weak / focal (when scattered positive cells are visible). ${ }^{[7]}$ This antibody stains nucleus in positive cells. Final diagnosis confirmed by clinicoradiological findings with histology and IHC.

\section{Statistical analysis}

Our study comprised 33 cases, of which 31 were PAs. One being a case of pituitary apoplexy, the final analysis was done on 31 cases of adenomas. Statistical analyses using SPSS software version 20.0 (IBM, Armonk, New York, USA) were used where appropriate for analysis and Prism GraphPad version 5 (GraphPad Software Inc., SanDiegoCalifornia Corporation,USA). $P<0.05$ was considered statistically significant.

\section{Results}

In our study, we have come across 31 cases of PA out of 33 cases, over a span of 2 years, at a tertiary super specialty hospital, which caters largely to the eastern part of India. Our patients ranged from 10 to 70 years,

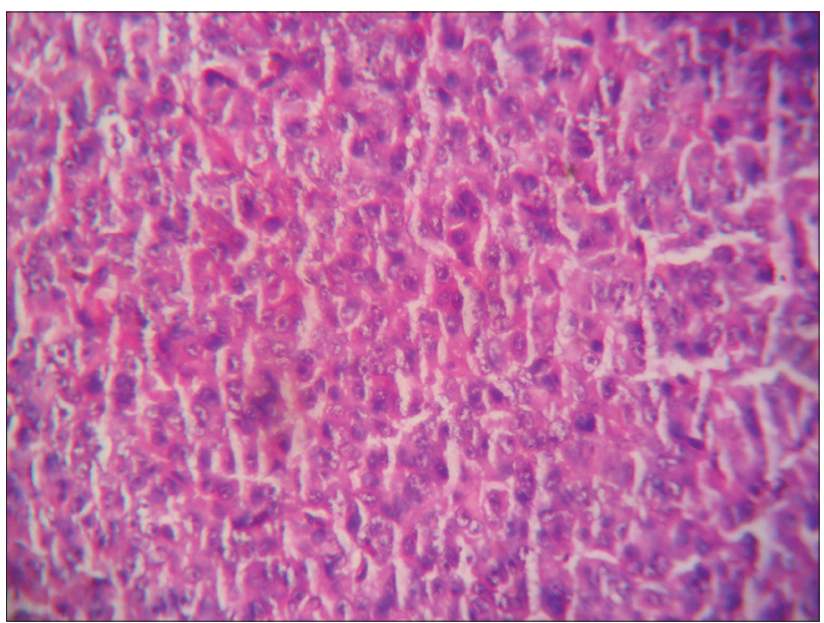

Figure 3: Amphophilic adenoma $\times 400$

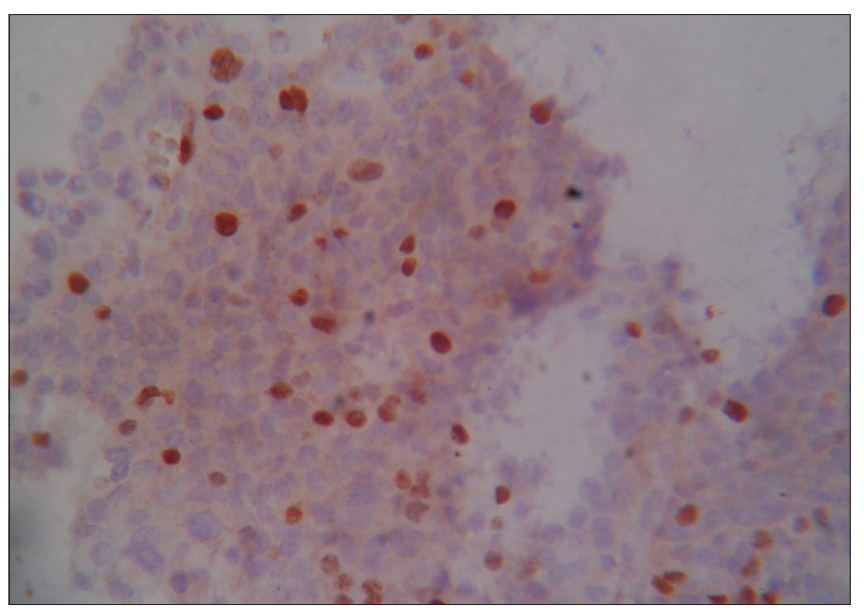

Figure 5: Ki-67 positive stain $\times 400$

being mainly in the $41-60$ years age group (48\%), with the mean age being 42.45 years \pm standard deviation (SD) 12.24. There was no significant sex predilection in our study (1:1.2). The other two cases which were not PA were noninfectious cysts - two cases of Rathke's cyst. $51 \%(16 / 31)$ patients presented with mass-related symptoms, such as headache and visual defects in our study. Next are the symptoms related to infertility mainly due to hyperprolactinemia 16\% (5/31). Patients presenting with acromegaly or Cushing`s symptoms were very few in our study total (15\%). Serum biochemical markers were raised in 15 of our patients, with PRL being the most common, seen in 10 out of 15 patients. Hence, in our study, we have found 51\% nonfunctioning pituitary adenoma (NFPA).

The radiology is of extreme importance in classifying a PA into a benign, atypical, or invasive PA. Radiology is useful in assessing the tumor size and tumor invasion. According to Knosp's classification radiologically, most common to occur were the macroadenomas, with cavernous sinus invasion (Grade IV). We have found 
$27 / 31$ cases with invasion in our study $(87 \%)$ and $4 / 31$ cases without invasion in our study (13\%), among them $2 / 31$ cases were noninvasive microadenoma. Other Grade III, Grade II, and Grade 0 comprised 16\%, 6\%, and $07 \%$ of all tumors, respectively. Grade I comprised no tumors in our study. Even though histological subtyping has become largely outdated, they have largely been superseded by IHC histology still gives us an idea about the subtype. So accordingly, in our study, acidophilic subtype is the most common (55\%), followed by the mixed subtype (19\%). The other subtypes basophilic and chromophobic are seen in 16\% and 7\% cases, respectively. We had 1 case of pituitary apoplexy which was excluded from the IHC tests; we conducted subsequently.

The IHC was conducted on 30 cases (excluding 1 case of pituitary apoplexy and 2 Rathke's cyst) where Ki-67, p53, and MGMT have been used for IHC in order to analyze the prognosis of the PA irrespective of the immunological subtype of the PA. In our study, only $13 \%$ (4 patients) had MGMT score 0 and 2 patients, among these 4 patients having above cutoff level of Ki-67 and p53 value considered as aggressive. Other score 1 , score 2, score 3 , score 4 , and score 5 comprised 20\% (6/30), 13\% (4/30), $10 \%(3 / 30), 17 \%(5 / 30)$ and $27 \%(8 / 30)$ of all tumors, respectively [Table 2].

Three percent is the cutoff between benign and atypical adenomas, with regard to Ki-67. In our study, only $13 \%$ (4 patients) were above 3\%. Further in p53 nuclear staining, $50 \%$ is considered as the cutoff between benign and atypical adenomas, and in our study, only $17 \%$ (five patients) were above $50 \%$.

Finally, combining the radiological Ki-67, p53, and MGMT values, two cases of aggressive adenoma have been seen in our study, the remaining being benign adenomas (WHO classification). We did not encounter any case of pituitary carcinoma. When comparing MGMT expression with recurrence, a high degree of significance was found (Mann-Whitney U-test, $P=0.0038$ ). Most of the recurrent tumors $(6 / 9)$ had MGMT score 1 or below and most of the nonrecurrent tumors had MGMT score 2 or above.

In our study, we found significant correlation between MGMT status with Knosp radiological grading (Mann-Whitney U-test, $P<0.0001$ ).When comparing MGMT expression with aggressiveness, a high degree of significance was found (Mann-Whitney U-test, $P<0.0001)$. All of our aggressive tumors $(2 / 30)$ had a MGMT score 0 , while most of the nonaggressive tumors (26/30) had a MGMT score 1 or above. In our study, we could not get any significant correlation between
Table 1: Scoring system for evaluation of 0-6-methylguanine-DNA methyltransferase immunostaining

\begin{tabular}{llcc}
\hline Score & \multicolumn{3}{c}{ Category } \\
\cline { 2 - 4 } & Distribution & Intensity & Percentage of positive cells \\
\hline 0 & No staining & - & - \\
1 & Heterogeneous & + & $<20$ \\
2 & Heterogeneous & $++/+++$ & $>20$ \\
3 & Homogenous & + & $<20$ \\
4 & Homogenous & $++/+++$ & $>20$ \\
5 & Homogenous & $++/+++$ & $>50$ \\
\hline$+:$ Mild, $++:$ Moderate,$+++:$ Strong staining
\end{tabular}

Table 2: Distribution of patients with respect to 0-6-methylguanine-DNA methyltransferase (score)

\begin{tabular}{lc}
\hline MGMT score & $\%$ of patients \\
\hline SCORE 0 & $13 \%$ \\
SCORE 1 & $20 \%$ \\
SCORE 2 & $13 \%$ \\
SCORE 3 & $10 \%$ \\
SCORE 4 & $17 \%$ \\
SCORE 5 & $27 \%$ \\
\hline
\end{tabular}

MGMT status histology where we found (Chi-square test, $P=0.488$ ) [Table 3].

When comparing Ki-67 expression with recurrence a high degree of significance was found (Mann-Whitney U-test, $P=0.0002)$. When comparing Ki-67 expression with aggressiveness, a high degree of significance was found (Mann-Whitney U-test, $P<0.0001$ ). All of our aggressive tumors (2/30) had a Ki-67 level $>3 \%$, while most of the nonaggressive tumors $(26 / 30)$ had a Ki-67 level $<3 \%$. When comparing p53 expression with aggressiveness, a high degree of significance was found (Chi-square test, $P=0.003$ ). All of our aggressive tumors $(2 / 30)$ had a p53 level $>50 \%$, while most of the nonaggressive tumors $(25 / 30)$ had a p53 level $<50 \%$.

\section{Discussion}

Clinically disturbing PA which are commonly detected are extremely rare with an estimated prevalence of $200 / 1,000,000$ and an incidence of 2/100,000/year. ${ }^{[8]}$ In our study too, over a span of 2 years, we found 33 cases of pituitary tumors, of which 31 turned out to be PAs. In a 10-year study conducted in Germany, Saeger et al. had published 4122 cases. ${ }^{[9]}$ Our study chiefly focused on the findings of one tertiary care center in the eastern part of India. Mahta et al. have done a study with 85 cases. $^{[10]}$ In India, Rishi et al. have conducted a study with 151 PA..$^{[1]}$ In the sellar region, a number of pathologies are common as highlighted by Lopes. ${ }^{[2]}$

We have found PAs to be $94 \%$ (total 31 cases among 33 cases) among our study cases. In our study, maximum 
Table 3: Correlation of 0-6-methylguanine-DNA methyltransferase with clinical parameters

\begin{tabular}{|c|c|c|c|c|c|c|c|c|}
\hline \multirow[t]{2}{*}{ Prognostic parameter } & \multirow[t]{2}{*}{ Number } & \multicolumn{6}{|c|}{ MGMT score } & \multirow[t]{2}{*}{$\boldsymbol{P}$} \\
\hline & & 0 & 1 & 2 & 3 & 4 & 5 & \\
\hline \multicolumn{9}{|l|}{ Age group } \\
\hline $1-20$ & 2 & 1 & 0 & 0 & 1 & 0 & 0 & \multirow[t]{4}{*}{0.343} \\
\hline $21-40$ & 11 & 1 & 2 & 2 & 1 & 2 & 3 & \\
\hline $41-60$ & 15 & 2 & 4 & 2 & 1 & 3 & 3 & \\
\hline $61-80$ & 2 & 0 & 0 & 0 & 0 & 0 & 2 & \\
\hline \multicolumn{9}{|l|}{ Histology } \\
\hline Basophilic & 5 & 2 & 0 & 0 & 1 & 0 & 2 & \multirow[t]{4}{*}{0.488} \\
\hline Chromophobic & 2 & 0 & 0 & 1 & 0 & 1 & 0 & \\
\hline Eosinophilic & 17 & 2 & 4 & 2 & 1 & 4 & 4 & \\
\hline Mixed & 6 & 0 & 2 & 1 & 1 & 0 & 2 & \\
\hline \multicolumn{9}{|l|}{ Aggressiveness } \\
\hline Nonaggressive & 28 & 2 & 6 & 4 & 3 & 5 & 8 & \multirow[t]{2}{*}{$<0.0001$} \\
\hline Aggressive & 2 & 2 & 0 & 0 & 0 & 0 & 0 & \\
\hline \multicolumn{9}{|l|}{ Recurrence } \\
\hline Recurrent & 9 & 1 & 5 & 2 & 0 & 1 & 0 & \multirow[t]{2}{*}{0.0038} \\
\hline Nonrecurrent & 21 & 3 & 1 & 2 & 3 & 4 & 8 & \\
\hline \multicolumn{9}{|c|}{ Knosp's Radiological grade } \\
\hline Grade 0 & 2 & 0 & 0 & 1 & 1 & 0 & 0 & \multirow[t]{4}{*}{0.608} \\
\hline Grade 2 & 2 & 0 & 0 & 0 & 0 & 1 & 1 & \\
\hline Grade 3 & 5 & 0 & 2 & 1 & 0 & 1 & 1 & \\
\hline Grade 4 & 21 & 4 & 4 & 2 & 2 & 3 & 6 & \\
\hline
\end{tabular}

MGMT=O-6-methylguanine-DNA methyltransferase

patients were found in the age distribution of 41-60 years age group, i.e., (15 patients) $48 \% .12$ patients (39\%) were in the 21-40 years age group, and two patients in the 1-20 years age group and two patients in the 61-80 years age interval. According to the $\mathrm{WHO}$, pituitary tumors are rarely seen in the pediatric population. ${ }^{[5]}$ We have also not encountered any patient in the pediatric age group in the course of our study. As highlighted by Kleinschmidt-DeMasters, the most common age group of PAs is 30-70 years and that corroborates with our findings. ${ }^{[12]}$ In a study by Rishi et al. also, $65 \%$ patients were between the ages of 30-60 years. ${ }^{[11]} 65 \%$ patients were aged between 40 and 70 years in our study as well.

The mean age of presentation in our study was 42.45 years \pm SD 12.24. The mean age of presentation was 41.6 years in a study by Mahta et al..$^{[10]}$ The WHO has not given a sex predilection in case of PAs. ${ }^{[5]}$ Our study also shows no significant distribution according to $\operatorname{sex}(\mathrm{M}: \mathrm{F}=1: 1.2)$. In a study by Kanter et al., PAs show a predilection for females in the pediatric age group, ${ }^{[13]}$ but in our study, we cannot encounter any pediatric patient. Rishi et al. have shown a male predominance among NFPAs. ${ }^{[1]}$

Most PA presented with mass effects, either singly or in combination with symptoms of hormone excess (25/31), and a few PA had only symptoms of hormone excess $(6 / 31)$. Many patients had an overlap of symptoms having both mass effects and symptoms of hormone excess (9/31) In study by Rishi et al., the most common presenting symptoms were visual symptoms and headache ${ }^{[11]}$ In our study, the functioning adenomas comprised GH-omas, PRL-omas, and ACTH-omas. PRL-omas (51\%) GH-omas are the most common and two patients have raised level of FSH. The other hormones, such as thyroid-stimulating hormone (TSH) and luteinizing hormone (LH), are not found to be raised in any of our patients. According to Osamura et al., frequent adenomas are $\mathrm{GH}$-omas and PRL-omas in their study. ${ }^{[14]}$ In a study by Ortiz-Plata et al., prolactinomas are most common $86 \%{ }^{[15]}$

According to Knosp's classification radiologically, most common to occur were the macroadenomas, with cavernous sinus invasion (Grade IV). We have found $27 / 31$ cases with invasion in our study $(87 \%)$ and $04 / 31$ cases without invasion in our study $(13 \%)$ among them $02 / 31$ cases were noninvasive microadenoma. A study by Ortiz-Plata et al. have found $18 \%$ cases belonging to Grade I and II, with the majority being Grade IV, ${ }^{[15]}$ this study corroborate with our study with Knosp's classification. In all cases in our study, the neuroradiological diagnosis was obtained using both contrast-enhanced brain computed tomography scan and magnetic resonance imaging.

Immunohistochemistry of the $30 \mathrm{PA}$ was done with Ki-67, p53, and MGMT. The immunoreactivity patterns of PA presented with specific features. MGMT shows both cytoplasm and nuclear positivity, but both Ki-67 and p53 show nuclear positivity. Accordingly 13\% (4/30) were adenomas having a MGMT score 0 and all of the aggressive adenomas (2/30) had MGMT score 0. A study by Dai et al. showed that MGMT ${ }^{[16]}$ reportedly counteracts the cytotoxic effects of the alkylating agent temozolomide. MGMT expression is often low in aggressive PAs and recurrent PAs.

Ki-67 (MIB-1 LI) was calculated using 3\% as the cutoff point between benign and atypical/invasive adenomas. Accordingly, 87\% (26/30) were benign adenomas and $13 \%$ (4/30) were adenomas having a MIB-1 LI of $>3 \%$. Saeger et al. showed a range of $0.16 \%-15.48 \% .{ }^{[9]}$ In our study, the upper limit did not exceed $10 \%$ and the range was $0.2 \%-10 \%$ The p53 was calculated using $50 \%$ as the cutoff point between benign and atypical/invasive adenomas. ${ }^{[7]}$ Accordingly, 83\% (25/30) were benign adenomas and $17 \%(5 / 30)$ were adenomas having a p53 level $\geq 50 \%$. In our study, the upper limit did not exceed $80 \%$ and the range was 3\%-80\% in our study. In a study by Saeger et al., p 53 expression has been found to correlate with MIB-1 labeling. ${ }^{[9]}$ In our study, these five patients who had p53 expression $\geq 50 \%$ among them four were seen to be Grade IV invasive tumors and among them two were seen aggressive tumors, having $>3 \%$ MIB- 1 LI. 
When comparing MGMT expression with aggressiveness, a high degree of significance was found (Mann-Whitney U-test, $P<0.0001)$ All of our aggressive tumors $(2 / 30)$ had a MGMT score 0 , while most of the nonaggressive tumors (26/30) had a MGMT score 1 or above. Further in our study, when comparing MGMT expression with recurrence, a high degree of significance was found (Mann-Whitney U-test, $P=0.0038$ ). Most of the recurrent tumors (6/9) had MGMT score 1 or below, and most of the nonrecurrent tumors had MGMT score 2 or above.

A study by Dai et al. showed that MGMT expression is not related to other clinicopathological indicators, such as invasiveness age, gender, tumor size, or functional status of patients with PAs. ${ }^{[16]}$ In our study, we could not get any significant correlation between MGMT status with age and histology $(P=0.343$ and 0.608 , respectively).

When comparing Ki-67 expression with aggressiveness, a high degree of significance was found (Chi-square test, $P=0.000)$. All of our aggressive tumors $(2 / 30)$ had a Ki-67 level $>3 \%$, while most of the nonaggressive tumors (26/30) had a Ki-67 level $<3 \%$. Thapar et al. ${ }^{[17]}$ reported that a $3 \% \mathrm{Ki}-67 \mathrm{LI}$ cutoff value is associated with $72.7 \%$ sensitivity and $97.3 \%$ specificity and a positive and negative predictive value of 96 and $80 \%$, respectively, in distinguishing noninvasive from invasive PAs. It has also been suggested that a Ki-67 LI $>10 \%$ should always raise suspicion regarding the malignant potential of the tumors. In the WHO classification, ${ }^{[5]}$ Ki-67 LI was regarded as a major predictive indicator for distinguishing benign from atypical adenomas. This corroborated with our study. Thus Ki-67 value is a good determinant of aggressiveness.

When comparing Ki-67 expression with Knosps radiological subtypes, no significance was found (Chi-square test, $P<0.414$ ). All of our Grade 0, Grade I, Grade II, and Grade III tumors had a Ki-67 level $<3 \%$, while majority of the Grade IV PA (17/21) had a Ki-67 level $<3 \%$ and only $(4 / 21)$ tumors had a Ki-67 level above $3 \%$. The study by Salehi et al. showed that the ability of Ki-67 LI to predict tumor invasiveness remains controversial, as discrepant results have been reported. ${ }^{[18]}$ In the study by Saeger et al., invasive PAs had a higher $\mathrm{Ki}-67$ value of $2.01 \% \pm 3.15 \%$ and noninvasive ones had a value of $1.12 \% \pm 1.87 \%{ }^{[9]}$ This was not corroborated with our study.

When comparing Ki-67 expression with histological subtypes a high degree of significance was found (Chi-square test, $P<0.005)$. Here, relatively high percentage of basophilic adenomas had a Ki-67 level above $3 \%$, whereas most of the other histological subtypes had a Ki-67 level below 3\%. Thus, histology is a good determinant of Ki-67 values. No significant relevant studies were available to criticize these findings.

When comparing p53 expression with aggressiveness, a high degree of significance was found (Chi-square test, $P=0.003)$. All of our aggressive tumors $(2 / 30)$ had a p53 level $>50 \%$, while most of the nonaggressive tumors $(25 / 30)$ had a p53 level $<50 \%$. In a study by Thapar et al., p53 immunoreactivity appears to correlate with tumor invasiveness, as it is present in $15 \%$ of invasive adenomas and all pituitary carcinomas but is absent in noninvasive adenomas; ${ }^{[19]}$ thus, p53 value is a good determinant of aggressiveness.

So coupled with the radiological findings of invasion and the immunohistological findings of MGMT score, Ki-67 $>3 \%$ and p53 $>50 \%$ immunostaining, $7 \%$ are aggressive adenomas, and the rest are benign adenomas according to WHO in our study. We have not received a single case of pituitary carcinoma. In the recent $\mathrm{WHO}$ classification, MGMT was regarded as a major predictive indicator for distinguishing benign from atypical adenomas. ${ }^{[20]}$ This corroborated with our study. Thus, MGMT value is a good determinant of aggressiveness and recurrence.

Two cases of invasive NFPAs with MIB- 1 LI of $50 \%-60 \%$ and $30 \%-40 \%$ p53 were seen in the study by Mahta et al..$^{[10]}$ Saeger et al. have reported $2.7 \%$ atypical adenomas and $0.12 \%$ pituitary carcinomas. ${ }^{[9]}$ Hence, atypical adenomas and pituitary carcinomas are extremely rare. We have had a very limited number of patients in this study, spanning 2 years. An increased number of cases will probably give us comparable data with the other studies pertaining to invasive adenomas.

We have the follow-up details of $48 \%$ of patients (15/14) of our study individuals over a span varying from 6 months to 2 years; one of our patients has died due to unrelated age-related complications, while another died due to unrelated other complications. The remaining $45 \%$ patients have been lost in follow-up.

\section{Conclusion}

PA is the most common pathology of the pituitary gland. The radiological grading, together with the prognostic markers of MGMT, Ki-67, and p53 are important markers which help in deciding the benign or atypical nature of the adenomas, thus helping in better patient management and predicting recurrences. Histopathology remains the gold standard in diagnosing and differentiating PA from other pathologies of the pituitary gland. Use of more immune markers and electron microscopy will help in subclassifying PA further. Temozolomide has demonstrated value in the treatment of aggressive PAs and carcinomas. The clinical and radiologic responses 
are encouraging. Clearly, there is an inverse correlation between MGMT immunoexpression and therapeutic response to temozolomide. Immunohistochemistry for MGMT appears to be a promising method for guiding therapeutic decision-making and predicting the therapeutic response to temozolomide. The molecular mechanisms that affect MGMT expression remain to be fully elucidated. Targeted modulation of MGMT may be useful in patients who otherwise may not respond to temozolomide therapy. Future therapies that change dosing regimens or that use pseudosubstrates may improve tumor responsiveness to temozolomide.

\section{Limitations}

1. Our cases are only 33, out of which PAs were 31 so over an increased span of time, we will be able to get more patients and that will give us a better idea about the behavior of PA

2. We have not got any case where the other hormones, such as TSH and LH, were raised, nor got cases of pituitary carcinomas, but with more number of patients we will probably get these presentations as well

3. Some patients have been lost in follow-up, so data about tumor behavior, like recurrences, have been lost too

4. Electron microscopy or cytogenetics was not done

5. Due to the limited time period of the study, follow-up of the patients treated with temozolomide and the correlation of the clinical outcome of the therapy with MGMT, Ki-67, and P53 status could not be evaluated.

\section{Financial support and sponsorship}

Nil.

\section{Conflicts of interest}

There are no conflicts of interest.

\section{References}

1. Gold EB. Epidemiology of pituitary adenomas. Epidemiol Rev 1981;3:163-83.

2. Lopes MB. Tumors of the pituitary gland. In: Fletcher CD, editor. Diagnostic Histopathology of Tumors. $4^{\text {th }}$ ed., Ch. 17. Philadelphia, PA, USA: Elsevier Saunders; 2013. p. 1146.

3. Patel YC, Ezzat S, Chik CL, Rorstad OP, Serri O, Ur E, et al. Guidelines for the diagnosis and treatment of acromegaly: A Canadian perspective. Clin Invest Med 2000;23:172-87.

4. Chatzellis E, Alexandraki KI, Androulakis II, Kaltsas G. Aggressive pituitary tumors. Neuroendocrinology 2015;101:87-104.

5. Lloyd RV, Kovacs K, Young WF Jr., Farrell WE, Asa SL, Trouillas J, et al. Tumours of the pituitary. In: DeLellis Ronald A, Lloyd RV,
Heitz Philipp U, Charis E, editors. WHO Classification of Tumours - Tumours of Endocrine Organs. $3^{\text {rd }}$ ed. France, Lyon: IARC Press; 2004. p. 12.

6. Mellai M, Caldera V, Annovazzi L, Chiò A, Lanotte M, Cassoni P, et al. MGMT promoter hypermethylation in a series of 104 glioblastomas. Cancer Genomics Proteomics 2009;6:219-27.

7. Hadzhiyanev A, Ivanova R, Nachev E, Elenkova A, Yaneva M, Zaharieva S, et al. Evaluation of prognostic utility of MIB-1 and p53 expression in pituitary adenomas: Correlations with clinical behaviour and follow-up results. Biotechnol Biotechnol Equip 2014;28:502-7.

8. Radhakrishnan K, Mokri B, Parisi JE, O'Fallon WM, Sunku J, Kurland LT. The trends in incidence of primary brain tumors in the population of Rochester, Minnesota. Ann Neurol 1995;37:67-73.

9. Saeger W, Lüdecke DK, Buchfelder M, Fahlbusch R, Quabbe HJ Petersenn S. Pathohistological classification of pituitary tumors: 10 years of experience with the German Pituitary Tumor Registry. Eur J Endocrinol 2007;156:203-16.

10. Mahta A, Haghpanah V, Lashkari A, Heshmat R, Larijani B, Tavangar SM. Non-functioning pituitary adenoma: Immunohistochemical analysis of 85 cases. Folia Neuropathol 2007;45:72-7.

11. Rishi A, Sharma MC, Sarkar C, Jain D, Singh M, Mahapatra AK, et al. A clinicopathological and immunohistochemical study of clinically non-functioning pituitary adenomas: a single institutional experience. Neurol India 2010;58:418-23.

12. Kleinschmidt-DeMasters BK. Pituitary gland. In: Rosai J, editor. Rosai and Ackerman's Surgical Pathology. 10 ${ }^{\text {th }}$ ed., Ch. 29. Milan, Italy: Elsevier Inc.; 2011. p. 2441-66.

13. Kanter SL, Mickle JP, Hunter SB, Rhoton AL. Pituitary adenomas in pediatric patients: Are they more invasive? Pediatr Neurosci 1985;12:202-4.

14. Osamura RY, Kajiya H, Takei M, Egashira N, Tobita M, Takekoshi S, et al. Pathology of the human pituitary adenomas. Histochem Cell Biol 2008;130:495-507.

15. Ortiz-Plata A, Tena-Suck ML, Pérez-Neri I, RembaoBojórquez D, Fernández A. Pituitary Adenomas - ClinicoPathological, Immunohistochemical and Ultrastructural Study.Adenomas P, Movaghar VR, Ed. 6, InTech. Chapter 4, Rijeka, Croatia 2012.

16. Dai C, Sun B, Liu X, Bao X, Feng M, Yao Y, et al. O-6-Methylguanine-DNA methyltransferase expression is associated with pituitary adenoma tumor recurrence: a systematic meta-analysis. Oncotarget 2017;8:19674-83.

17. Thapar K, Kovacs K, Scheithauer BW, Stefaneanu L, Horvath E, Pernicone PJ, et al. Proliferative activity and invasiveness among pituitary adenomas and carcinomas: An analysis using the MIB-1 antibody. Neurosurgery 1996;38:99-106.

18. Salehi F, Agur A, Scheithauer BW, Kovacs K, Lloyd RV, Cusimano M. Ki-67 in pituitary neoplasms: A review - Part I. Neurosurgery 2009;65:429-37.

19. Thapar K, Scheithauer BW, Kovacs K, Pernicone PJ, Laws ER Jr. P53 expression in pituitary adenomas and carcinomas: Correlation with invasiveness and tumor growth fractions. Neurosurgery 1996;38:765-70.

20. Lloyd RV, Osamura Robert Y. Tumours of the pituitary. In: Kloppel G, Rosai J, editors. WHO Classification of Tumours - Tumours of Endocrine Organs. $4^{\text {th }}$ ed. France, Lyon: IARC Press; 2017. p. 18. 